\title{
Experiences of racial discrimination and cardiometabolic risk among
}

\section{Australian children}

\author{
Naomi Priest ${ }^{\mathrm{a}, \mathrm{b}}$, Mandy Truong ${ }^{\mathrm{a}, \mathrm{b}}$, Shiau Chong ${ }^{\mathrm{a}, \mathrm{b}}$, Yin Paradies ${ }^{\mathrm{c}}$, Tania L King ${ }^{\mathrm{d}}$, Anne \\ Kavanagh $^{\mathrm{d}}$, Tim Olds ${ }^{\mathrm{e}}$, Jeffrey M Craig ${ }^{\mathrm{f}}$, David Burgner ${ }^{\mathrm{g}, \mathrm{h}, \mathrm{i}}$.
}

a ANU Centre for Social Research and Methods, Australian National University, Canberra, Australia

b Population Health, Murdoch Children's Research Institute, Parkville, Australia

c Faculty of Arts and Education, Deakin University, Burwood, Australia

d Disability and Health Unit, Centre for Health Equity, Melbourne School of Population and Global Health, University of Melbourne, Carlton, Australia

e Alliance for Research in Exercise Nutrition and Activity (ARENA), Sansom Institute, School of Health Sciences, University of South Australia, Adelaide, Australia

f Centre for Molecular and Medical Research, Deakin University School of Medicine, Geelong, Australia

g Infection and Immunity, Murdoch Children's Research Institute, Royal Children's Hospital, Australia

h Department of Paediatrics, Melbourne University, Parkville, Australia

i Department of Paediatrics, Monash University, Clayton, Australia

\begin{abstract}
Cardiometabolic disease is a leading cause of adult morbidity and mortality globally. There is considerable evidence that childhood adversity is associated with markers of cardiometabolic disease risk in childhood, including obesity, blood pressure trajectories, and chronic inflammation. Experiences of racial discrimination may be an important, yet under explored, form of childhood adversity influencing childhood cardiometabolic risk. This study aimed to examine associations between self-reported racial discrimination and cardiometabolic risk markers among children. A total of 124 children ( 73 female) aged 11.4 years (SD 0.71)
\end{abstract}


participated in the study. Most children ( $\mathrm{n}=79)$ identified as being from an Indigenous or an ethnic minority background. Markers of cardiometabolic risk were BMI, waist circumference, weight height ratio, systolic and diastolic blood pressure, and five inflammatory markers (C-reactive protein (CRP), Interleukin (IL)-1 $\beta$, IL-6, IL-8, and TNF$\alpha)$. Results showed that two or more reported experiences of racial discrimination were associated with increased BMI z-score (b $0.58,95 \%$ CI 0.18, 0.99), waist circumference (b $4.91 \mathrm{~cm}, 95 \%$ CI $0.71,9.1$ ), systolic blood pressure (b 2.07mmHg, 95\% CI $0.43,3.71$ ) and IL6 (b $0.13,95 \%$ CI $0.00,0.27)$ and marginally associated with TNF- $\alpha$ (b $0.22,95 \%$ CI -0.09 , $0.54)$ after adjusting for socio-demographic covariates. Findings from this study suggest the need to address racism and racial discrimination as important social determinants of cardiometabolic risk and of the inequitable burden of cardiometabolic disease experienced by those from Indigenous and minoritized ethnic backgrounds.

Key words discrimination, children, inflammation, cardiometabolic, adversity, ethnicity 


\section{Introduction}

Cardiometabolic disease is a leading cause of adult morbidity and mortality globally, but disease risk accrues across the life course. Socioeconomic and racial ethnic inequalities in the incidence, treatment and outcomes of cardiometabolic disorders are also widely documented. ${ }^{1,2}$ Yet there are important but often over-looked social determinants of cardiometabolic risk. ${ }^{3,4}$ A critical determinant of health and health inequalities for both children and adults is racial discrimination, defined as differential or unfair treatment due to actual or perceived membership of a racial or ethnic group. ${ }^{5,6}$ Evidence across contexts and groups highlights the relationships between experiences of racial discrimination and mental health and behavioural difficulties among children and adolescents. ${ }^{5}$

There is considerable evidence that childhood adversity is associated with markers of cardiometabolic disease risk in childhood, such as obesity ${ }^{7}$, blood pressure trajectories ${ }^{8}$ and chronic inflammation. ${ }^{9,10}$ Experiences of racial discrimination may be an important, yet under explored, form of childhood adversity influencing childhood cardiometabolic risk. ${ }^{5,11}$

Childhood adversity may influence cardiometabolic outcomes via behavioural, mental health and biological pathways. ${ }^{11}$ Behaviours such as sleep, physical activity and diet are all poorer following adversity. ${ }^{11}$ Mental health problems can be initiated or intensified by adversity, predisposing children and adolescents to early cardiometabolic risk. ${ }^{11}$ In addition, due to disruption of many physiological regulatory processes, childhood adversity can directly affect biological processes. ${ }^{11}$ These putative mechanisms interact and influence other cardiometabolic factors such as blood pressure and adiposity, and subsequently influence cardiometabolic morbidity and mortality throughout the life course. ${ }^{11}$

Experiences of racial discrimination have been associated with a range of cardiometabolic risk markers and outcomes among adults, including hypertension, carotid intima media 
thickness (a preclinical atherosclerosis phenotype), coronary artery calcification, blood pressure, adiposity and chronic inflammation. ${ }^{12}$ Yet associations between racial discrimination experiences and cardiometabolic outcomes at earlier ages remain largely unexamined, except in two studies of African Americans in the United States, only one of which reports outcomes pre-adulthood. One small cross-sectional study ( $\mathrm{n}=42)$ found associations between experiences of racial discrimination and C-reactive protein (CRP), a marker of chronic inflammation, and systolic and diastolic blood pressure among 10-15 year olds. ${ }^{13}$ Another study $(n=160)$ found experiences of racial discrimination between ages 17-19 years were associated with increased cytokine levels at 22 years. ${ }^{14}$ To our knowledge there are no previous studies of self-reported experiences of racial/ethnic discrimination in relation to cardiometabolic risk markers among ethnic minority youth outside of the United States, and none anywhere that consider measures of obesity, central adiposity, blood pressure and multiple inflammatory markers.

Using data from the Speak Out Against Racism (SOAR) study, we examined associations between self-reported experiences of racial/ethnic discrimination and cardiometabolic risk markers, including BMI, waist circumference, weight height ratio, systolic and diastolic blood pressure, and inflammation (CRP, Interleukin (IL)-1 $\beta$, IL-6, IL-8, and TNF- $\alpha$ ). We hypothesised that racial discrimination would be associated with increased BMI, waist circumference, waist height ratio, blood pressure and inflammatory biomarkers.

\section{Methods}

\section{Participants and procedures}

Data were drawn from the Speak Out Against Racism (SOAR) study, a large-scale, population representative cross-sectional study of government school students in Years 5-9 (10-15 years of age) in the states of New South Wales (NSW) and Victoria (VIC), Australia. 
SOAR collected data on students' experiences of, attitudes and responses to, and impacts on health, wellbeing and educational outcomes, of racism and racial bullying. Full details are documented elsewhere, but in brief, researchers visited school classrooms and administered surveys either online or on paper as negotiated with individual schools. ${ }^{5}$ Schools chose their preferred mode of survey delivery after they had agreed to participate. Three primary schools in Victoria agreed to participate in a sub-study in which anthropometrics, blood pressure and inflammatory markers were also collected from students in Years 5-6 (10-12 years of age) following parental consent and student assent. Schools with high proportions of students from Indigenous and ethnic minority backgrounds were invited to participate in the sub-study study given the focus on racial discrimination experiences. Students from Indigenous and ethnic minority backgrounds are more likely to attend disadvantaged schools. As such the three participating schools were all below average socio-educational advantage as measured using the Index of Community Socio-Educational Advantage (ICSEA). ICSEA is a composite of average parent occupation and education across students, school geographic location, and proportion of Indigenous students; higher ICSEA corresponds to higher socioeducational advantage at the school level. ${ }^{15}$ The participating schools all had very similar ICSEA (948-970) with the Victorian state average ICSEA equal to 1031, and nationally an ICSEA of between 975-1024 is considered average level of advantage. Trained researchers visited schools and provided students with an interactive tutorial about how scientists measure what happens 'under the skin' and to explain the data collection processes in order to build interest in science and minimise any discomfort before data collection. ${ }^{16}$ The tutorial introduction broadly discussed that the overall study aim was to understand student's experiences of racial discrimination and how this might be impacting them, however most of the tutorial focused on how scientists can measure what happens in the body. All data collection occurred between 9.15 am and 12 noon to allow for diurnal patterns of 
inflammatory markers. Ethics approval was obtained from the Australian National University and from the Department of Education and Training Victoria and permission was obtained from each participating school principal.

\section{Measures}

\section{Racial discrimination experiences}

Students reported if they had ever experienced racial discrimination using 10 items drawn from the Adolescent Discrimination Distress Index (ADDI) ${ }^{17}$ together with two items used previously with diverse Australian school students. ${ }^{18}$ Four items assessed discrimination by peers at school (e.g. did other students leave you out of their activities), three items assessed discrimination by school personnel (e.g. were you put in a lower ability class or group) and five items assessed discrimination by societal perpetrators (e.g. were you treated unfairly by a shop assistant or security guard). Each discrimination item was followed by the attribution 'because of your race/ethnicity/cultural background'. While culture is not synonymous with race or ethnicity, in Australia culture is commonly used as a proxy for, and conflated with, race and/or ethnicity and so was included, following the precedent of previous studies. ${ }^{18}$ Students responded to each item as 'this did not happen to me', 'once or twice', 'every few weeks', 'about once a week', or 'several times a week or more'. Following previous approaches ${ }^{19}$ each item was coded as a binary variable (did not happen to me vs. once or twice/every few weeks/once a week/several times a week) and the binary variables were then summed. A binary indicator variable was then created from this summed variable (two or more vs one or no experiences) following common approaches in the childhood adversity literature..$^{20}$

Body Mass Index (BMI) 
Students' height was measured to the nearest $0.1 \mathrm{~cm}$ using a portable rigid Seca stadiometer and their weight to the nearest $0.1 \mathrm{~kg}$ using Seca 803 digital scales. Participants were asked to remove shoes and heavy clothing items. Body Mass Index (BMI) was calculated as weight $(\mathrm{kg}) /$ height $\left(\mathrm{m}^{2}\right)$. Age and sex specific BMI z-scores were created based on the Centers for Disease Control (CDC) growth charts. ${ }^{21}$

\section{Waist circumference}

Waist circumference was measured using a flexible tape to the nearest $1 \mathrm{~mm}$ at the midpoint between the lower ribs and the pelvic bone. Three measurements were taken and the mean of these three measures was used for analysis.

\section{Waist-height ratio}

Waist-height ratio (WHtR) was calculated as waist circumference $(\mathrm{cm}) /$ height $(\mathrm{cm})$.

\section{Blood pressure}

Systolic and diastolic blood pressure were measured using an A\&D Medical 1010 automatic inflation blood pressure monitor while students were seated at a table with both feet flat on the ground and their arm supported. Three measures were taken with one minute timed rest in between and the second two measures were averaged and used in analysis.

\section{Inflammatory markers}

Whole saliva samples were collected using the passive drool method ${ }^{22}$ and stored at $-80^{\circ} \mathrm{C}$ until assayed. Saliva was assayed for CRP and for IL-1 $\beta$, IL-6, TNF- $\alpha$, and IL-8. Saliva samples were thawed and batch-analysed using a commercially available ELISA assay (Salimetrics, USA) according to manufacturer's instructions. Thawed samples were centrifuged at $1500 \mathrm{x} g$ for $15 \mathrm{~min}$ to produce cell-free saliva and this was used without 
further processing for all assays. All samples were brought to room temperature before adding to the assay wells and two technical replicates were averaged. The test sensitivity was $10 \mathrm{pg} / \mathrm{mL}$ for CRP, and for IL-1 $\beta$, IL-6, IL-8, and TNF- $\alpha 0.07 \mathrm{pg} / \mathrm{mL}, 64 \mathrm{pg} / \mathrm{mL},<0.37$ $\mathrm{pg} / \mathrm{mL}$, and $0.106 \mathrm{pg} / \mathrm{mL}$ respectively.

\section{Covariates}

Ethnicity was measured using a self-reported variable using categories developed for the study. Self-reported race/ethnicity (with the exception of Indigenous status) is not routinely collected in national Australian data collections so a standard classification is not available. Students were able to select multiple racial/ethnic categories and an open-ended 'other' category was available that was later back-coded. Following international approaches, ${ }^{23} \mathrm{a}$ prioritisation method was used to classify multiple responses to mutually exclusive categories based on level of stigmatisation in Australia. ${ }^{24}$ Due to the small sample size in this sub-study, a binary ethnicity variable was created (Anglo/European [White] vs Indigenous/ethnic minority) for analytic models. ${ }^{25}$ Students self-reported country of birth (coded as born overseas/born in Australia), gender (boy, girl, other) and age. Oral lesions, medication use, and recent or current illness were also self-reported by students as salivary biomarkers may be affected by these factors. but in this sample there was no statistical evidence that these illness and medication factors were associated with any of the salivary biomarkers, they were not included in further analysis.

\section{Analysis plan}

Salivary cytokines (IL-1 $\beta$, IL-6, IL-8, and TNF- $\alpha$ ) variables were positively skewed and natural log-transformed to produce approximately normal distributions and normal residuals in the regression models. Multiple linear regression models with robust standard estimation to 
adjust for clustering by school were run separately for each of the cardiometabolic outcomes to estimate the association between racial discrimination and each outcome. In Model 1, we adjusted for ethnicity, age, gender and country of birth. The model for BMI was not adjusted for age and sex as these variables were used to generate BMI z-scores.

There were very small missing data $(\mathrm{N}=4)$ on each of the anthropometric and blood pressure outcomes and a higher amount of missing data on the inflammatory markers $(\mathrm{N}=30$ for $\mathrm{CRP}$ and between $\mathrm{N}=18-20$ for cytokines). Ethics approval processes required parental consent to be obtained separately for anthropometric measures, blood pressure and collection of saliva samples with lower response rates for saliva collection accounting for higher levels of missingness. Variations in sample size for each of the salivary biomarkers were artefacts of laboratory processing. There was no statistical evidence that missingness varied by ethnicity, country of birth or gender. Complete case analysis was used for each of the separate multiple logistic regression models given potential for bias using multiple imputation with small sample sizes and non-normally distributed variables (inflammatory outcomes). ${ }^{26}$ It is also suggested that there is much less to be gained from multiple imputation when missingness is in the outcome of interest than when missingness is in covariates used to control for confounding ${ }^{27}$. As such multiple imputation in this present study was considered likely to increase rather than reduce the potential for biased estimates and was not used.

\section{Results}

Study sample characteristics are shown in Table $1.21 \%$ of eligible students in schools agreed to participate in the biomarker sub-study. Students had a mean age of 11.4 years (SD 0.71) and slightly more of the sample were female (59\%). The study sample was highly diverse, with 36\% students identifying as Anglo/European and most students (64\%) from an Indigenous or an ethnic minority background. In this latter group, $15 \%$ were 
Indigenous/Pacific Islander/Maori, 18\% Middle Eastern/African/Latin American (68\% Middle Eastern, most (80\%) of whom identified as Afghani), 20\% South Asian, and 11\% South East/East Asian. As $\leq 2$ participants identified as Indigenous, Latin American and East Asian these groups were combined with larger groups to maintain confidentiality and sample size although in doing so do not wish to confuse or conflate the unique identities of these groups. Thirty percent of students were born overseas with almost all (93\%) of the Anglo/Euro participants born in Australia compared to over half (56\%) of the Indigenous/Ethnic Minority participants (50\% of South Asian and South East/East Asian; 27\% of Middle Eastern/African/Latin American; 44\% Indigenous/Pacific Islander/Maori). Countries of birth were Afghanistan, Bangladesh, Cambodia, Oman, Pakistan, Samoa, South Sudan, Sri Lanka, India, Philippines, Malaysia, Bosnia, Fiji, New Zealand and the United States).

Over a third (35\%) of the students reported two or more experiences of racial discrimination. Nearly half (47\%) of the Indigenous/Ethnic Minority group (including Pacific Islander/Maori, Middle Eastern, African, Latin American, South Asian and South East Asian) had two or more experiences of racial discrimination compared to $13 \%$ among Anglo/European students.

Table 2 shows associations between racial discrimination and cardiometabolic risk markers. Two or more reported experiences of racial discrimination were associated with increased BMI z-score (b 0.58, 95\% CI 0.18, 0.99), waist circumference (b $4.91 \mathrm{~cm}, 95 \%$ CI 0.71, 9.1), systolic blood pressure (b 2.07mmHg, 95\% CI 0.43, 3.71) and IL-6 (b 0.13, 95\% CI 0.00, 0.27 ) and marginally associated with TNF- $\alpha$ (b $0.22,95 \%$ CI $-0.09,0.54)$ after adjusting for socio-demographic covariates.

\section{Discussion}


This study aimed to examine associations between self-reported experiences of racial/ethnic discrimination and cardiometabolic risk markers, including BMI, waist circumference, systolic and diastolic blood pressure, and inflammation (CRP, IL-1 $\beta$, IL-6, IL-8, and TNF$\alpha$ ), among diverse Australian children. In a unique contribution to the literature, findings show the harmful effects of racial/ethnic discrimination experiences on child cardiometabolic risk markers, including BMI, waist circumference, systolic blood pressure and salivary IL-6. There was weak evidence of an association between racial/ethnic discrimination and TNF- $\alpha$. We found evidence that racial/ethnic discrimination was associated with markers of obesity and central adiposity, that is, BMI and waist circumference. This is consistent with extant evidence of the harmful effects of racial discrimination on obesity risk among children and adolescents ${ }^{28,29}$ and the larger body of work among adults. ${ }^{30,31}$ The difference in BMI z-score associated with two or more racial/ethnic discrimination experiences is over half a standard deviation above the CDC age- and sex-specific mean scores and likely to have considerable clinical impact. Waist circumference was also associated with racial discrimination experiences. The difference of $4.91 \mathrm{~cm}$ represents considerable clinical impact, although confidence intervals around this estimate were wide $(95 \%$ CI $0.71-9.1)$. The lack of evidence for an association between racial discrimination and weight height ratio likely reflects the smaller variance in weight height ratio compared to waist circumference, with more research needed using larger samples within and across age groups among children from Indigenous and from ethnic minoritized groups.

We also found evidence of an association between racial/ethnic discrimination experiences and systolic blood pressure. This is consistent with one small previous study conducted in the US among 24 African American and white 10-15 year olds. ${ }^{13}$ In contrast with this previous study, we did not find racial/ethnic discrimination associated with diastolic blood pressure. 
Among adults associations between self-reported racial/ethnic discrimination and hypertension and resting blood pressure measures are mixed, ${ }^{30,32}$ with suggestions that associations may be gender specific and influenced by psychosocial processes related to individual coping styles, racial/ethnic identity and interpretations of what is fair or unjust. It is plausible that the interrelatedness of these psychosocial processes with associations between self-reported racial/ethnic discrimination experiences and blood pressure may be even more salient during childhood and adolescence when physiological and psychosocial systems are highly sensitive and developing rapidly.

A further contribution of this study are the findings that experiences of racial/ethnic discrimination were associated with elevated IL-6, but not with CRP or other inflammatory cytokines. The association between racial/ethnic discrimination and IL-6 remained after adjusting for BMI. This is counter to a previous study that found racial/ethnic discrimination was associated with CRP among 10-15 year olds in the US, although this study did not measure inflammatory cytokines. ${ }^{13}$ Another US study among late adolescents reported associations between racial discrimination and increased cytokine levels using a composite measure but did not report results for individual cytokines. ${ }^{14}$ Maternal experiences of racial/ethnic discrimination have also been associated with levels of salivary IL-6 (but not cytokines) among children aged 4-9 years in a small (N=54) US study ${ }^{33}$ and with hsCRP among 8-16 year old Hispanic/Latino Youth in the US. ${ }^{34}$ More broadly, while relatively few studies have examined associations between childhood adversity and child and adolescent inflammation, findings to date suggest a trend towards positive findings ${ }^{35}$ One large scale longitudinal prospective study found acute adverse events in middle childhood (occurring between ages 6 to 8), as well as cumulative adversity from birth to 8 years, were associated with higher levels of IL-6 and CRP at age $10{ }^{36}$

Commented [NP1]: Reviewer comment: The authors found differences for different outcomes, particularly for the inflammatory markers. What might these differences tell you about the mechanisms involved? Linked to this I also felt a discussion about what each of the inflammatory markers in particular, but also the other outcomes, captures and their relationships with stressful experiences more broadly. Playing Devil's advocate - why include all these markers and not just one - what does each tell you? 
This study's findings that children's racial discrimination experiences are associated with multiple cardiometabolic risk markers in childhood including overweight and obesity markers, blood pressure and some inflammatory markers, are consistent with concepts of allostasis and allostatic load. These concepts highlight that stressor exposure initiates highly integrated responses across nervous, endocrine, and immune systems with ongoing activation of these systems implicated in both short and long term consequences of stressor exposure in childhood.$^{37}$ For example, increased overweight and obesity risk in response to stress such as discrimination experiences may be related to structural and functional changes in the nervous system that can lead to behavioural responses including physical activity participation and eating patterns, as well as to endocrine responses resulting from chronic activation of the HPA axis including elevated cortisol levels and development of glucocorticoid resistance. ${ }^{37}$ Increased systolic blood pressure may be related to repeated or chronic activation of the sympathetic nervous system and down regulation of the parasympathetic nervous system. ${ }^{37}$ Increased inflammation is also likely related to changes in sympathetic and parasympathetic nervous system regulation. The findings of this present study showed different associations between racial discrimination and different inflammatory markers, with evidence of association with IL-6 and marginally for TNF-alpha but not for CRP or other inflammatory cytokines. IL-6 is upstream to CRP. As CRP is an acute phase reactant, it may not be an optimal marker of chronic inflammation in childhood. ${ }^{38}$ The findings may also reflect limitations of using salivary CRP as an biomarker of systemic (rather than intra-oral) inflammation; use of saliva was a pragmatic choice as blood sampling was not possible. Further, each of these five inflammatory markers can be associated with both acute and chronic inflammatory responses, and may be elevated in response to both alloimmune (nonself) and autoimmune threats. ${ }^{39}$ But they have different kinetics, different associations with disease states (e.g. TNF-alpha is associated with major depression, IL-8 and IL-6 with 
obesity). ${ }^{39}$ Therefore, it is potentially more informative to measure a wide range of inflammatory biomarkers markers.

\section{Limitations}

This analysis is cross-sectional. It is possible that children and adolescents who are overweight or obese may be more likely to report discrimination experiences, ${ }^{28}$ in which case the omission of BMI as a confounder in analytical models may have biased estimates.

However, longitudinal studies suggest racial discrimination precedes overweight and obesity, in which case overweight or obesity status mediates the relationship between racial discrimination and cardiometabolic outcomes. ${ }^{28} \mathrm{We}$ considered this to be the more plausible pathway, thus it's inclusion in models would bias estimates. Given this, we did not include BMI in the main analyses. Salivary cytokines may partly reflect local intra-oral inflammation. However, they are considered indicative of chronic systemic inflammation ${ }^{40}$ and correlate with serum levels in response to psychosocial stress. ${ }^{41,42}$ Saliva samples have been found previously to be valid for CRP. ${ }^{43,44}$ While salivary cytokine levels may partly reflect local intra-oral inflammation, they are considered indicative of chronic systemic inflammation ${ }^{40}$ and have been shown to correlate with serum levels in response to psychosocial stress. ${ }^{41}$ Compared to blood, saliva samples are quick, simple and non-invasive and more amenable to collection in non-clinical settings such as schools. ${ }^{22,41}$ Racial discrimination experiences were self-reported and some measurement error is likely. Although a three-minute rest prior to blood pressure measurement was not strictly enforced, findings are consistent with previous research. The use of a school level measure of socioeducational advantage rather than directly measuring family socioeconomic position may introduce unmeasured confounding and influence findings. However, child report of family 
socioeconomic position is not reliable and parental report was not possible in this study. Longitudinal analyses across larger samples with measurement of a broader range of confounders would provide better evidence of causal relations and allow for more robust examination of potential mediating effects of BMI on associations between racial/ethnic discrimination and inflammatory outcomes. Collection of blood samples to allow analysis of biomarkers not possible in saliva as well as broader measures of cardiovascular risk phenotypes and adiposity beyond anthropometrics are also important extensions to this study. However, these findings among a sample of ethnically diverse children are an important contribution to a field of research where little is known and few datasets contain the required information for this type of investigation.

Findings from this study suggest the need to address racism and racial discrimination as important social determinants of cardiometabolic risk and of the inequitable burden of cardiometabolic disease experienced by those from Indigenous and minoritized ethnic backgrounds. Preventing and countering racism and racial discrimination across school and educational settings, as well as throughout societal institutions and systems via context specific interventions, together with policy and practice reforms are critical public health and clinical priorities. $^{45,46}$

\section{Conclusion}

This study among an ethnically diverse, socio-educationally disadvantaged sample of Australian children found two or more experiences of racial/ethnic discrimination were associated with increased BMI, waist circumference, systolic blood pressure, and IL-6 and weakly associated with TNF- $\alpha$. Racism and racial discrimination are critically important to tackle as critical social determinants of cardiometabolic risk for children and adolescents. 


\section{Acknowledgements}

We wish to thank the staff and students from the schools that participated in the study. This

work was supported by an Australian Research Council Linkage Project Grant (LP140100413);

an Australian National University Research School of Social Sciences Cross-college grant; and funding from the School of Social Sciences and Psychology at Western Sydney University.

The first author is supported by a National Health and Medical Research Council Career Development Fellowship (APP1123677).

\section{References}

1. Havranek EP, Mujahid MS, Barr DA, et al. Social Determinants of Risk and Outcomes for Cardiovascular Disease: A Scientific Statement From the American Heart Association. Circulation 2015; 132(9): 873-98.

2. Stringhini S, Carmeli C, Jokela M, et al. Socioeconomic status and the $25 \times 25$ risk factors as determinants of premature mortality: a multicohort study and meta-analysis of 1.7 million men and women. Lancet 2017; 389(10075): 1229-37.

3. Camhi SM, Katzmarzyk PT. Tracking of cardiometabolic risk factor clustering from childhood to adulthood. International Journal of Pediatric Obesity 2010; 5(2): 122-9.

4. McGill HC, Jr., McMahan CA, Herderick EE, Malcom GT, Tracy RE, Strong JP. Origin of atherosclerosis in childhood and adolescence. Am J Clin Nutr 2000; 72(5 Suppl): 1307s-15s.

5. Priest N, Paradies Y, Trenerry B, Truong M, Karlsen S, Kelly Y. A systematic review of studies examining the relationship between reported racism and health and wellbeing for children and young people. Social Science and Medicine 2013; 95: 115-27.

6. Paradies $\mathrm{Y}$, Ben J, Denson N, et al. Racism as a Determinant of Health: A Systematic Review and Meta-Analysis. PLoS One 2015; 10(9): e0138511.

7. Elsenburg LK, van Wijk KJ, Liefbroer AC, Smidt N. Accumulation of adverse childhood events and overweight in children: A systematic review and meta-analysis. Obesity 2017; 25(5): 820-32.

8. Su S, Wang X, Pollock JS, et al. Adverse childhood experiences and blood pressure trajectories from childhood to young adulthood: the Georgia stress and Heart study. Circulation 2015; 131(19): 1674-81.

9. Slopen N, Goodman E, Koenen KC, Kubzansky LD. Socioeconomic and other social stressors and biomarkers of cardiometabolic risk in youth: a systematic review of less studied risk factors. PLoS One 2013; 8(7): 1-12.

10. Slopen N, Koenen K, Kubzansky LD. Childhood adversity and immune and inflammatory biomarkers associated with cardiovascular risk in youth: A systematic review. Brain, Behavior, and Immunity 2012; 26: 239-50.

11. Suglia SF, Koenen KC, Boynton-Jarrett R, et al. Childhood and adolescent adversity and cardiometabolic outcomes: a scientific statement from the American Heart Association. Circulation 2018; 137(5): e15-e28. 
12. Lewis TT, Cogburn CD, Williams DR. Self-reported experiences of discrimination and health: scientific advances, ongoing controversies, and emerging issues. Annu Rev Clin Psychol 2015; 11: 407-40.

13. Goosby BJ, Malone S, Richardson EA, Cheadle JE, Williams DT. Perceived discrimination and markers of cardiovascular risk among low-income African American youth. American Journal of Human Biology 2015; 27(4): 546-52.

14. Brody GH, Yu T, Miller GE, Chen E. Discrimination, racial identity, and cytokine levels among African-American adolescents. Journal of Adolescent Health 2015; 56(5): 496-501.

15. Australia Curriculum AaRA. What does the ICSEA value mean? ; 2015.

16. Truong M, Sharif M, Moorhead R, et al. Using biosocial methods in child health disparities research in Australian primary schools. under review.

17. Fisher CB, Wallace SA, Fenton RE. Discrimination distress during adolescence. Journal of Youth \& Adolescence 2000; 29(6): 679-95.

18. Priest N, Perry R, Ferdinand A, Paradies Y, Kelaher M. Experiences of racism, racial/ethnic attitudes, motivated fairness and mental health outcomes among primary and secondary school students. J Youth Adolescence 2014; 43: 1672-87.

19. Benner $A D$, Graham $S$. The antecedents and consequences of racial/ethnic discrimination during adolescence: does the source of discrimination matter? Dev Psychol 2013; 49(8): 1602-13.

20. Bethell CD, Carle A, Hudziak J, et al. Methods to Assess Adverse Childhood Experiences of Children and Families: Toward Approaches to Promote Child Well-being in Policy and Practice. Acad Pediatr 2017; 17(7s): S51-s69.

21. Ogden C, Carroll M, Kit B, Flegal K. Prevalence of obesity and trends in Body Mass Index among US children and adolescents, 1999-2010. JAMA 2012; 307(5): 483-90.

22. Granger DA, Kivlighan KT, Fortunato C, et al. Integration of salivary biomarkers into developmental and behaviorally-oriented research: problems and solutions for collecting specimens. Physiology \& behavior 2007; 92(4): 583-90.

23. Cormack D, Robson C. Classification and output of multiple ethnicities: issues for monitoring Māori health. Wellington, 2010.

24. Priest N, Chong S, Truong M, et al. Findings from the 2017 Speak Out Against Racism (SOAR) student and staff surveys. CSRM working paper no. 3/2019. Canberra: ANU Centre for Social Research \& Methods, Australian National University, 2019.

25. Priest N, Chong S, Truong M, et al. Findings from the 2017 Speak Out Against Racism (SOAR) student and staff surveys. Canberra: Centre for Social Research and Methods, Australian National University, 2019.

26. Lee K, Carlin J. Multiple imputation in the presence of non-normal data. Statistics in Medicine 2016; 36: 606-17.

27. Lee KJ, Carlin JB. Recovery of information from multiple imputation: a simulation study. Emerging themes in epidemiology 2012; 9(1): 3.

28. Priest N, Becares L, Kavanagh A, King T. Cumulative Effects of Bullying and Racial Discrimination on Adolescent Health in Australia. Journal of Health and Social Behavior 2019; 60(3): 344-

61.

29. Chambers EC, Tull ES, Fraser HS, Mutunhu NR, Sobers N, Niles E. The relationship of internalized racism to body fat distribution and insulin resistance among African adolescent youth. $J$ Nat/Med Assoc 2004; 96(12): 1594-8.

30. Lewis T, Wiliams DR, Tamene M, Clark C. Self-Reported Experiences of Discrimination and Cardiovascular Disease. Curr Cardiovasc Risk Rep 2014; 1(8): 365.

31. Bernardo CO. Interpersonal discrimination and markers of adiposity in longitudinal studies: a systematic review. Obesity Review 2017; 18(9): 1040-9. 
32. Brondolo E, Pencille M, Schoenthaler A, Ogedegbe G. Racism and Hypertension: A Review of the Empirical Evidence and Implications for Clinical Practice. American Journal of Hypertension 2011; online march 2011.

33. Condon EM, Holland ML, Slade A, Redeker NS, Mayes LC, Sadler LS. Associations Between Maternal Experiences of Discrimination and Biomarkers of Toxic Stress in School-Aged Children. Maternal and Child Health Journal 2019: 1-5.

34. Slopen N, Strizich G, Hua S, et al. Maternal experiences of ethnic discrimination and child cardiometabolic outcomes in the Study of Latino Youth. Annals of epidemiology 2019.

35. Slopen N, Koenen KC, Kubzansky LD. Childhood adversity and immune and inflammatory biomarkersassociated with cardiovascular risk in youth: A systematic review. Brain, Behavior, and Immunity 2012; 26: 239-50.

36. Slopen N, Kubzansky LD, McLaughlin KA, Koenen KC. Childhood adversity and inflammatory processes in youth: a prospective study. Psychoneuroendocrinology 2012; 38: 188.

37. Danese A, McEwen B. Adverse childhood experiences, allostasis, allostatic load, and agerelated disease. Physiology and Behaviour 2012; 106: 29-39.

38. Collier F, Ellul S, Juonala M, et al. Glycoprotein acetyls (GlycA) at 12 months are associated with high-sensitivity C-reactive protein and early life inflammatory immune measures. Pediatric Research 2019; 85(5): 584-5.

39. Chen L, Deng H, Cui H, et al. Inflammatory responses and inflammation-associated diseases in organs. Oncotarget 2017; 9(6): 7204-18.

40. Condon EM. Chronic Stress in Children and Adolescents: A Review of Biomarkers for Use in Pediatric Research. Biol Res Nurs 2018; 20(5): 473-96.

41. La Fratta I, Tatangelo R, Campagna G, et al. The plasmatic and salivary levels of IL-1 $\beta$, IL-18 and IL-6 are associated to emotional difference during stress in young male. Scientific Reports 2018; 8(1): 3031.

42. Riis JL, Granger DA, Minkowitz CS, Bandeen-Roche K, DiPietro JA, Johnson SB. Maternal distress and child neuroendocrine and immune regulation. Social Science \& Medicine 2016; 151: 206-14.

43. Out D, Hall RJ, Granger DA, Page GG, Woods SJ. Assessing salivary C-reactive protein: longitudinal associations with systemic inflammation and cardiovascular disease risk in women exposed to intimate partner violence. Brain, behavior, and immunity 2012; 26(4): 543-51.

44. Slavish DC, Graham-Engeland JE, Smyth JM, Engeland CG. Salivary markers of inflammation in response to acute stress. Brain, behavior, and immunity 2015; 44: 253-69.

45. Trent M, Dooley DG, Dougé J. The impact of racism on child and adolescent health Pediatrics 2019; 144(2): e20191765.

46. Rutland A, Killen M. A Developmental Science Approach to Reducing Prejudice and Social Exclusion: Intergroup Processes, Social-Cognitive Development, and Moral Reasoning. Social Issues and Policy Review 2015; 9(1): 121-54. 